\title{
(GIGA)bYte
}

\section{Chromosome-level genome assembly of the humpback puffer, Tetraodon palembangensis}

Rui Zhang1, ${ }^{1, \dagger}$ Chang Li ${ }^{1, \dagger}$, Mengjun $\mathrm{Yu}^{1, \dagger}$, Xiaoyun Huang ${ }^{1}$, Mengqi Zhang ${ }^{1}$, Shanshan Liu ${ }^{1}$, Shanshan Pan ${ }^{1}$, Weizhen Xue ${ }^{1}$, Congyan Wang ${ }^{1}$, Chunyan $\mathrm{MaO}^{1}$, He Zhang ${ }^{1,2, *}$ and Guangyi Fan ${ }^{1, *}$

1 BGI-Qingdao, BGI-Shenzhen, Qingdao 266555, China

2 Department of Biology, Hong Kong Baptist University, Hong Kong, China

\section{ABSTRACT}

The humpback puffer, Tetraodon palembangensis, is a poisonous freshwater pufferfish species mainly distributed in Southeast Asia (Thailand, Laos, Malaysia and Indonesia). The humpback puffer has many interesting biological features, such as inactivity, tetrodotoxin production and body expansion. Here, we report the first chromosome-level genome assembly of the humpback puffer. The genome size is $362 \mathrm{Mb}$, with a contig N50 value of $\sim 1.78 \mathrm{Mb}$ and a scaffold N50 value of $\sim 15.8 \mathrm{Mb}$. Based on this genome assembly, $\sim 61.5 \mathrm{Mb}(18.11 \%)$ repeat sequences were identified, 19,925 genes were annotated, and the function of $90.01 \%$ of these genes could be predicted. Finally, a phylogenetic tree of ten teleost fish species was constructed. This analysis suggests that the humpback puffer and T. nigroviridis share a common ancestor 18.1 million years ago (MYA), and diverged from T. rubripes 45.8 MYA. The humpback puffer genome will be a valuable genomic resource to illustrate possible mechanisms of tetrodotoxin synthesis and tolerance.

Subjects Animal and Plant Sciences, Animal Genetics, Functional Genomics, Marine Biology

Submitted: $\quad 05$ November 2020

Accepted: $\quad 31$ March 2021

Published: $\quad 01$ April 2021

* Corresponding authors. E-mail: zhanghe@genomics.cn;

fanguangyi@genomics.cn

$\dagger$ Contributed equally.

Published by GigaScience Press.

Preprint submitted at

https://www.preprints.org/

manuscript/202008.0694/v1

This is an Open Access article distributed under the terms of the Creative Commons Attribution License (http://creativecommons.org/ licenses/by/4.0/), which permits unrestricted reuse, distribution, and reproduction in any medium, provided the original work is properly cited.

Gigabyte, 2021, 1-12

\section{DATA DESCRIPTION}

\section{Background and context}

The humpback puffer, Tetraodon palembangensis (NCBI Taxonomy ID: 1820603, Fishbase ID: 25179), also known as Pao palembangensis, is widely distributed in Southeast Asia and prefers to live in alkalescent, warm $\left(24-28^{\circ}\right)$, and slow-flowing rivers (Figure 1a) [1]. The female and male humpback puffers have a similar body size, but the male's rear hump is much bigger than that of the female [2]. The humpback puffer is a popular ornamental fish because of its beautiful skin colouration and patterns. Unlike other species of predatory pufferfish, the humpback puffer is lazy and will not initiatively look for food [1]. Furthermore, its body contains a deadly toxin, known as tetrodotoxin (TTX), and it can swell up to three times its normal size as a defense mechanism when threatened [1]. Previous studies have shown that the toxicity of the humpback puffer varies greatly between seasons [3]. The wild population of humpback puffer has declined in recent years owing to the destruction of suitable habitat caused by climate change and overfishing [4].

With these biological characteristics and a small genome size, the humpback puffer is an ideal species for genetic study [5]. It is also a species in the Fish10K Genome Project, a subproject of the Earth BioGenome Project, which aims to sample, sequence, assemble and analyse the genomes of 10,000 fish species [6]. In this study, we provide a chromosome-scale 
a

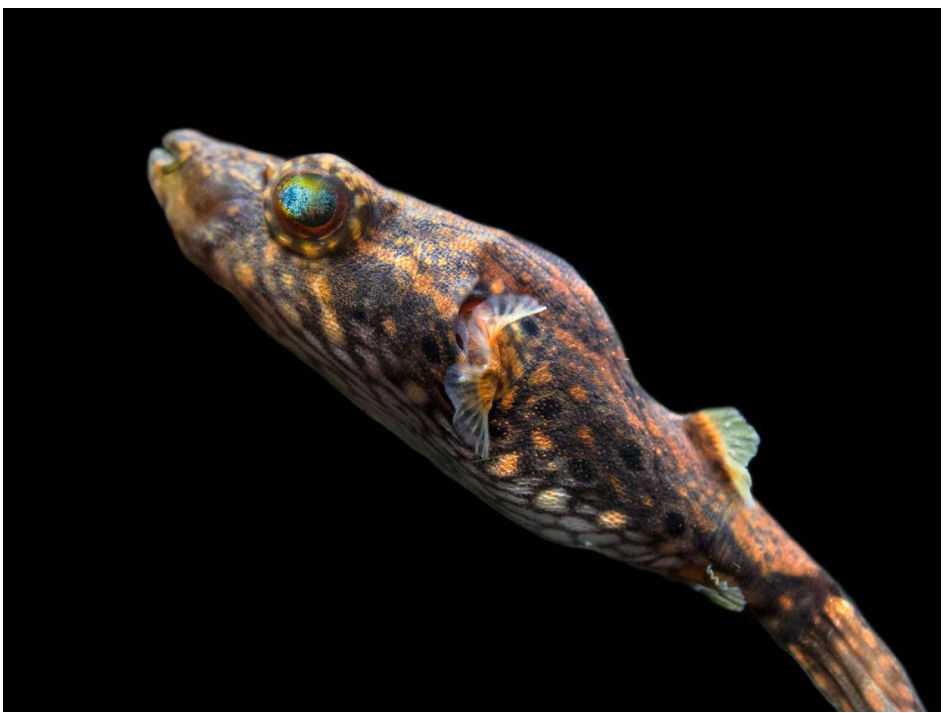

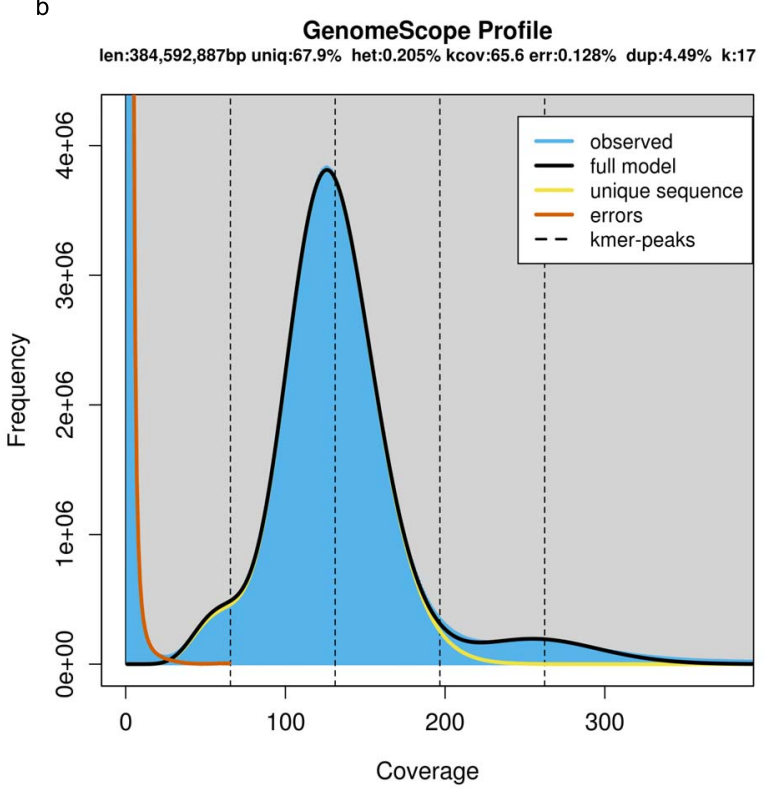

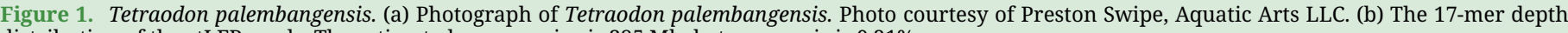
distribution of the stLFR reads. The estimated genome size is $385 \mathrm{Mb}$; heterozygosis is $0.21 \%$.

genome assembly of the humpback puffer. This assembly will be valuable for further study of mechanisms, such as tetrodotoxin synthesis and expansion defense. Comparative genomic analysis will help us to better understand the phylogenetic evolution and special gene families of the Tetraodontidae.

\section{METHODS}

All methods used to isolate DNA/RNA, construct libraries, and conduct genomic sequencing are available in a protocols.io collection (Figure 2 [7]).

\section{Sample collection and sequencing}

The sample (CNGB ID: CNS0224034) used in this study was an adult humpback puffer bought from the YueHe Flower-Bird-Fish market in Guangzhou Province, China. DNA and RNA were both isolated from blood following published protocols [8, 9]. Then, a paired-end single tube long fragment reads (stLFR) library and an RNA library were constructed according to the protocol published by Wang et al. [10]. A Hi-C library was constructed from blood according to the protocol published by Huang et al. [11]. These three libraries were then sequenced on the BGISEQ-500 platform (RRID:SCR_017979) [12]. A Nanopore library was constructed with DNA isolated from blood using the QIAamp DNA Mini Kit (Qiagen) [13] and sequenced on the GridION platform (RRID:SCR_017986) [14]. In total, we obtained $146 \mathrm{~Gb}(\sim 312 \times)$ raw stLFR data, 21 Gb raw RNA data, $19 \mathrm{~GB}(\sim 49 \times)$ raw Hi-C data, and 12 GB $(\sim 32 \times)$ raw Nanopore data (Table 1$)$.

Raw stLFR reads were subjected to quality control to improve the assembly quality. Firstly, we obtained co-barcoding information from the last 42 bases of read 1 and deleted the last 42 bases. Then SOAPnuke (v1.6.5, RRID:SCR_015025) [15] was used to filter remaining reads, using the parameters: “-M 1 -d -A 0.4-n $0.05-\mathrm{l} 10-\mathrm{q} 0.4-\mathrm{Q} 2-\mathrm{G}-5$ 0" [16]. 


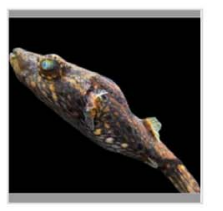

Mar 24, 2021

is Bookmark

Copy / Fork
(1) Protocols for "Chromosome-level genome assembly of the humpback puffer, Tetraodon palembangensis"

Rui Zhang ${ }^{1}$, Chang Li ${ }^{1}$, Mengjun Yu ${ }^{1}$, Xiaoyun Huang ${ }^{1}$, Mengqi Zhang ${ }^{1}$, Shanshan Liu', Shanshan Pan ${ }^{1}$, Weizhen Xue ${ }^{1}$, Congyan Wang ${ }^{1}$, Chunyan Mao ${ }^{1}$, He Zhang ${ }^{2}$, Guangyi Fan ${ }^{1}$

${ }^{1} \mathrm{BGI}-$ Qingdao, BGI-Shenzhen, Qingdao 266555, China;

${ }^{2}$ BGI-Qingdao, BGI-Shenzhen, Qingdao 266555, China.Department of Biology, Hong Kong Baptist Univ ersity, Hong Kong, China

Works for me dx.doi.org/10.17504/protocols.io.bs8inhue

BGI GIGA 1 more workspace

$\therefore$ zhangrui7

Figure 2. Protocols.io collection for the chromosome-level genome assembly of the humpback puffer, Tetraodon palembangensis. https://www.protocols.io/widgets/doi?uri=dx.doi.org/10.17504/protocols.io.bs8inhue

\begin{tabular}{|c|c|c|c|c|c|}
\hline \multirow[t]{2}{*}{ Libraries } & \multirow[t]{2}{*}{ Read lengths (bp) } & \multicolumn{2}{|c|}{ Raw data } & \multicolumn{2}{|c|}{ Valid data } \\
\hline & & Total bases (Gb) & $\begin{array}{l}\text { Sequencing } \\
\text { depth }(x)\end{array}$ & Total bases (Gb) & $\begin{array}{c}\text { Sequencing } \\
\text { depth }(x)\end{array}$ \\
\hline stFLR & PE100 & 120.4 & 311.69 & 62.6 & 162.60 \\
\hline RNA & PE 100 & 20.6 & - & 20.0 & - \\
\hline $\mathrm{Hi}-\mathrm{C}$ & PE 100 & 19.01 & 49.43 & 5.4 & 14.04 \\
\hline Nanopore & CN50: $32 \mathrm{~kb}$ & 12.3 & 31.98 & - & - \\
\hline
\end{tabular}

Table 2. Statistics of 17-mer analysis.

\begin{tabular}{ccccc}
\hline $\boldsymbol{k}$-mer & $\boldsymbol{k}$-mer number & $\boldsymbol{k}$-mer depth $(\times)$ & Heterozygosity (\%) & Genome size (bp) \\
\hline 17 & $48,458,703,762$ & 126 & 0.205 & $384,592,887$ \\
\hline
\end{tabular}

Finally, $62 \mathrm{~Gb}(152 \times)$ clean data were retained for further assembly. Raw RNA reads were also filtered by SOAPnuke using the parameters: “-M 1 -A 0.4-n $0.05-\mathrm{l} 10-\mathrm{q} 0.4-\mathrm{Q} 2-\mathrm{G}-5$ 0 ”, generating $20 \mathrm{~Gb}$ clean data. Raw Hi-C data were produced with a quality control using HiC-Pro (v. 2.8.0) [16] with default parameters. This generated $5.4 \mathrm{~Gb}$ validated data, which accounted for $28.81 \%$ of all data (Table 1 ).

\section{Genome assembly}

Jellyfish (v2.2.6, RRID:SCR_005491) was used to count $k$-17mers of all clean stLFR reads [17]. Genomescope [18] was used to estimate the humpback puffer genome size at about $385 \mathrm{Mb}$ (Table 2 and Figure 1b).

The genome size, $G$, was defined as $G=K_{\text {num }} / K_{\text {depth }}$, where the $K_{\text {num }}$ is the total number of $k$-mers, and $K_{\text {depth }}$ is the most frequently occurring frequency.

To assemble the humpback puffer genome, we firstly converted the format of clean stLFR reads, then used Supernova (v. 2.0.1, RRID:SCR_016756) to perform the draft assembly. 


\begin{tabular}{ccc}
\hline Table 3. Statistics of the draft assembly of the humpback puffer genome. \\
\hline Statistics & Scaffold & Contig \\
Total number (\#) & 5291 & 6190 \\
Total length (bp) & $361,704,206$ & $360,427,744$ \\
Gap (N) (bp) & $1,276,462$ & 0 \\
Average length (bp) & 68,362 & 58,227 \\
N50 length (bp) & $7,059,990$ & $1,830,664$ \\
N90 length (bp) & 453,057 & 157,209 \\
Maximum length (bp) & $19,534,197$ & $9,842,180$ \\
Minimum length (bp) & 6882 & 48 \\
GC content (\%) & 44.66 & 44.66 \\
\hline
\end{tabular}

\begin{tabular}{ccc}
\hline Table 4. Statistics of the Hi-C scaffolding of the humpback puffer genome. & \\
\hline Statistics & Scaffold & Contig \\
Total number (\#) & 5366 & 6435 \\
Total length (bp) & $361,698,760$ & $360,427,744$ \\
Gap (N) (bp) & $1,271,016$ & 0 \\
Average length (bp) & 67,406 & 56,011 \\
N50 length (bp) & $15,808,960$ & $1,794,775$ \\
N90 length (bp) & $11,014,520$ & 117,115 \\
Maximum length (bp) & $34,916,285$ & $9,792,502$ \\
Minimum length (bp) & 682 & 48 \\
GC content (\%) & 44.66 & 44.66 \\
\hline
\end{tabular}

Then, we used GapCloser (v. 1.12, RRID:SCR_015026) [19] to fill gaps with stLFR reads. To futher improve the assembly quality, TGSgapFiller [20] was then used to re-fill gaps with Nanopore reads, and Pilon (v. 1.22, RRID:SCR_014731) [21] was used to polish the assembly twice. At this stage, the genome assembly was about $362 \mathrm{Mb}$, with 7.1-Mb scaffold N50 and 1.8-Mb contig N50 values (Table 3).

With the genome and validated Hi-C data from HiC-Pro, the contact matrix was generated by Juicer (v3, RRID:SCR_017226). Finally, we perfomed chromosomal-level scaffolding using the 3D de novo assembly (3D-DNA) pipeline (v. 170123) [22]. This anchored $91.2 \%$ of all sequences to 18 chromosomes, with a length ranging from $11 \mathrm{Mb}$ to $35 \mathrm{Mb}$ (Table 4 and Figure 3).

The karyotype differs among genuses in Tetraodontidae [23-27]. For example, Takifugu rubripes, Takifugu obscurus and Takifugu flavidus have $2 n=44$ chromosomes, while Tetraodon nigroviridis and Tetraodon fluviatilis have $2 n=42$ chromosomes. In addition, Thamnaconus septentrionalis (Monacanthidae) has $2 n=40$ chromosomes. Thus, we defined the chromosome number of the humpback puffer to be 18, according to the apparent and logical interactions by Hi-C reads.

\section{Genomic annotation}

Two methods were used to annotate repetitive sequences. Firstly, we aligned the genome to the Repbase library by TRF (v.4.09) [28]. RepeatMasker (v. 3.3.0, RRID:SCR_012954) and RepeatProteinMask (v. 3.3.0) [29] were then used to predict and classify the repetitive sequences. Secondly, we constructed a repeat library using RepeatModeler (v1.0.8, RRID:SCR_015027) and classified the transposable elements (TEs) with RepeatMasker (v. 3.3.0) [29]. The results of both methods were amalgamated to give a total of $65 \mathrm{Mb}$ repeat sequences and $59 \mathrm{Mb}$ TEs, accounting for $18.11 \%$ (Table 5 and Figure $4 \mathrm{a}$ ) and $16.62 \%$ of the entire genome, respectively (Table 6 and Figure $4 \mathrm{a}$ ). 


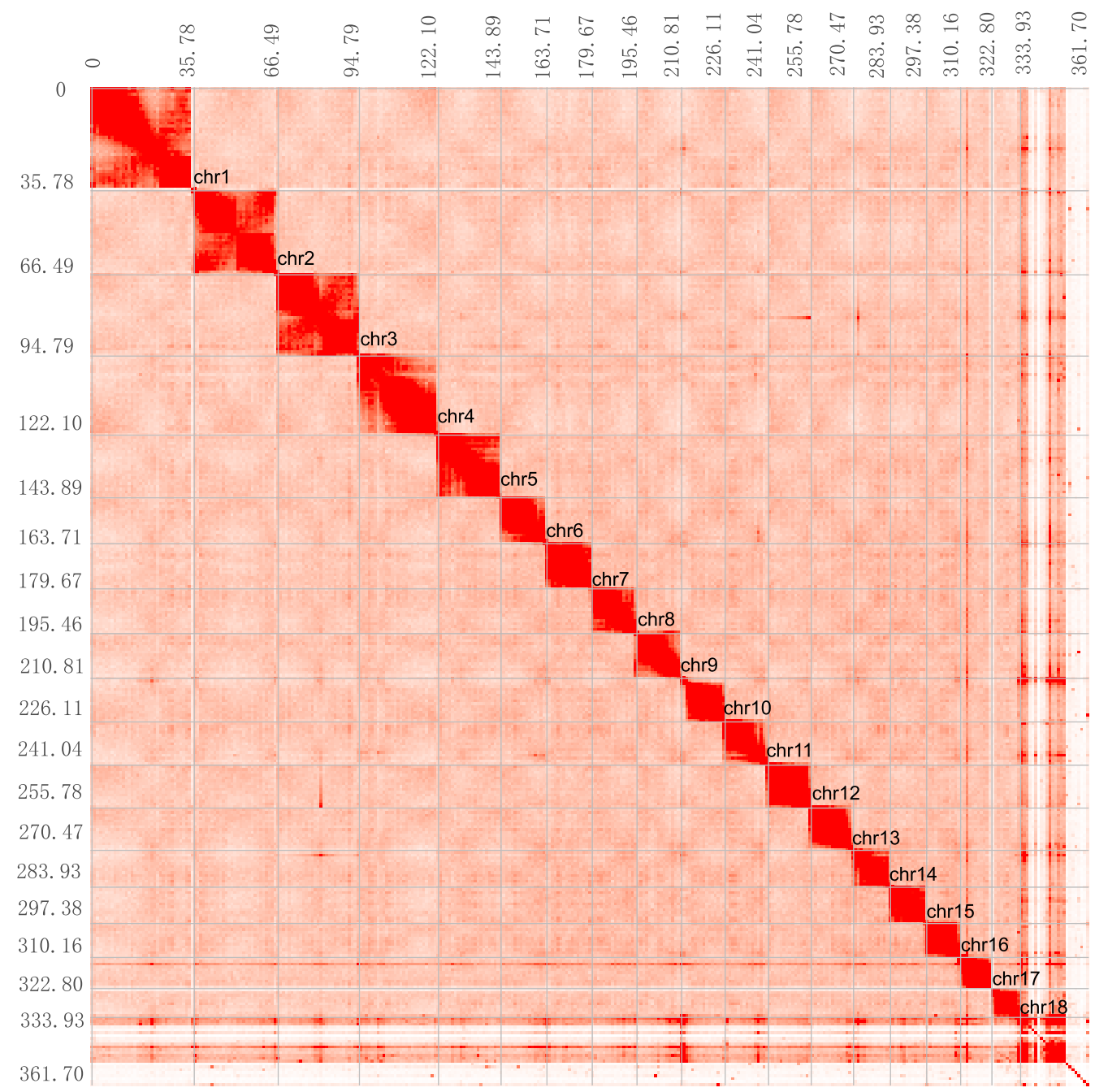

Figure 3. Heat map of chromosomal interaction from Hi-C reads. Grey lines show the border between chromosomes.

Using the clean, reformatted stLFR reads, the mitochondrial genome of the humpback puffer was assembled using MitoZ [30] with default parameters. Mitochondrial genes were annotated using the MitoAnnotator tool of the mitofish pipeline [31] (Figure 4b). For gene structural annotation, we performed de novo prediction using AUGUSTUS (v3.1, RRID:SCR_008417) [32], GlimmerHMM (v3.0.4, RRID:SCR_002654) [33], and Genscan (RRID:SCR_013362) [34]. We also used TRINITY (v2.8.5, RRID:SCR_013048) [35] to assemble a draft transcriptome with clean RNA reads, then HISAT2 (v2.1.0, RRID:SCR_015530)-StringTie (v1.3.4, RRID:SCR_016323) [36] and PASA (v2.3.3, RRID:SCR_014656)-TransDecoder (RRID:SCR_017647) [37] to predict transcripts. GeneWise (v2.4.1, RRID:SCR_015054) [38] was used for homologous annotation, with protein data obtained from the National Center for Biotechnology Information (NCBI) database for the following eight species: Danio rerio (NCBI, GenBank ID:50), Cynoglossus semilaevis (NCBI, GenBank ID:11788), Gasterosteus aculeatus (NCBI, GenBank ID:146), Gadus morhua (NCBI, GenBank ID:2661), Larimichthys 

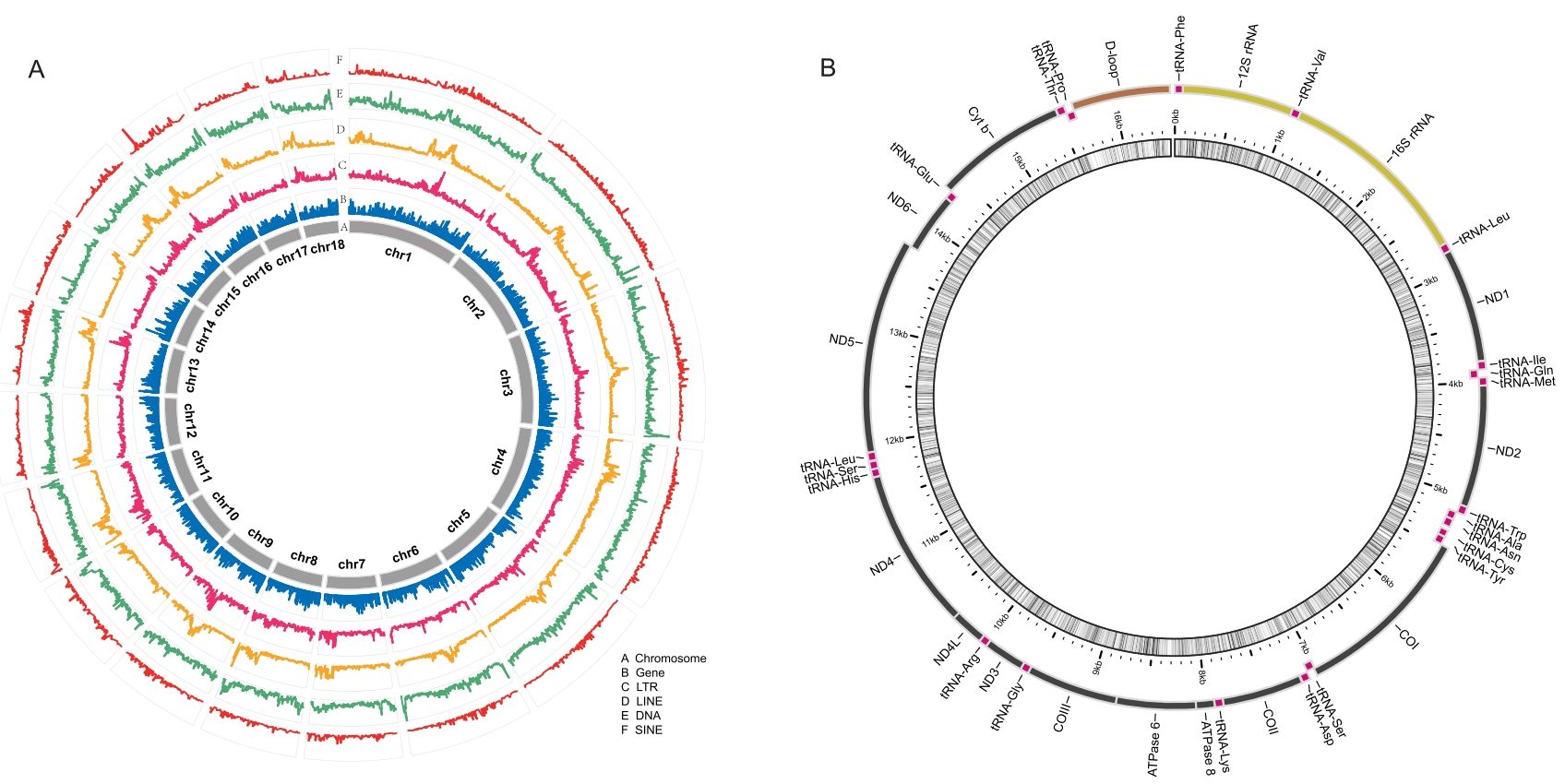

Figure 4. Annotation of the Tetraodon palembangensis genome. (a) Basic genomic elements of the genome. LTR, long terminal repeat; LINE, long interspersed nuclear elements; SINE, short interspersed elements. (b) Physical map of mitochondrial assembly.

\begin{tabular}{ccc|}
\hline Table 5. Statistics of repeat sequences. & \\
\hline Type & Repeat size (bp) & \% of genome \\
TRF & $9,050,571$ & 2.52 \\
RepeatMasker & $34,142,529$ & 9.50 \\
RepeatProteinMask & $17,674,660$ & 4.92 \\
\hline De novo & $57,492,865$ & 16.00 \\
Total & $65,080,476$ & 18.11 \\
\hline
\end{tabular}

crocea (NCBI, GenBank ID:12197), Oreochromis niloticus (NCBI, GenBank ID:197), Oryzias latipes (NCBI, GenBank ID:542), and Takifugu rubripes (NCBI, GenBank ID:63). Finally, these three types of evidence were integrated using EVidenceModeler (v1.1.1,

RRID:SCR_014659) [39], generating 19,925 nonredundant coding genes, each containing an average of 11 exons and a 1945 bp coding region (Table 7).

For gene function annotation, we aligned the 19,925 genes to the TrEMBL (UniProtKB, RRID:SCR_004426) [40], Swissprot [41], Kyoto Enyclopedia of Genes and Genomes (KEGG, RRID:SCR_012773) [42], Gene Ontology (GO, RRID:SCR_002811) [43] and InterProScan (RRID:SCR_005829) [44] databases. Overall, 90.1\% of all genes were able to be functionally annotated (Table 8 and Figure 3).

\section{Genome evolution}

To study the evolutionary status of humpback puffer among bony fish species, we clustered gene families by alignment using protein sequences of the humpback puffer and nine other teleosts (Xiphophorus maculatus, Gasterosteus aculeatus, Sebastes schlegelii, Oryzias latipes, Gadus morhua, Oreochromis niloticus, Tetraodon nigroviridis, Danio rerio, and Takifugu rubripes) using the TreeFam v0.50 pipeline [45]. Protein-coding genes sequences for all of 


\begin{tabular}{|c|c|c|c|c|c|c|c|c|}
\hline \multirow[t]{2}{*}{ Type } & \multicolumn{2}{|c|}{ RepBase TEs } & \multicolumn{2}{|c|}{ TE proteins } & \multicolumn{2}{|c|}{ De novo } & \multicolumn{2}{|c|}{ Combined TEs } \\
\hline & Length (bp) & $\%$ of genome & Length (bp) & $\%$ of genome & Length (bp) & $\%$ of genome & Length (bp) & $\%$ of genome \\
\hline DNA & $12,412,491$ & 3.45 & $1,086,262$ & 0.30 & $16,089,219$ & 4.48 & $22,470,373$ & 6.25 \\
\hline LINE & $18,430,929$ & 5.13 & $13,695,154$ & 3.81 & $29,418,621$ & 8.19 & $33,421,782$ & 9.30 \\
\hline SINE & 524,061 & 0.15 & 0 & 0.00 & 289,252 & 0.08 & 789,086 & 0.22 \\
\hline LTR & $5,393,600$ & 1.50 & $2,906,451$ & 0.81 & $12,758,934$ & 3.55 & $15,803,098$ & 4.40 \\
\hline Other & 8,290 & 0.00 & 228 & 0.00 & 0 & 0.00 & 8,518 & 0.00 \\
\hline Unknown & 0 & 0.00 & 0 & 0.00 & $3,202,764$ & 0.89 & $3,202,764$ & 0.89 \\
\hline Total & $34,142,529$ & 9.50 & $17,674,660$ & 4.92 & $55,052,617$ & 15.32 & $59,729,335$ & 16.62 \\
\hline
\end{tabular}

\begin{tabular}{|c|c|c|c|c|c|c|c|}
\hline & Gene set & Gene number & $\begin{array}{c}\text { Average } \\
\text { transcript } \\
\text { length (bp) }\end{array}$ & $\begin{array}{l}\text { Average CDS } \\
\text { length (bp) }\end{array}$ & $\begin{array}{l}\text { Average intron } \\
\text { length (bp) }\end{array}$ & $\begin{array}{l}\text { Average exon } \\
\text { length (bp) }\end{array}$ & $\begin{array}{l}\text { Average exons } \\
\text { per gene }\end{array}$ \\
\hline \multirow[t]{8}{*}{ Homolog } & Cynoglossus semilaevis & 19,686 & 9136.12 & 1715.14 & 856.1 & 177.4 & 9.67 \\
\hline & Danio rerio & 19,348 & $15,066.80$ & 1577.39 & 1718.92 & 178.28 & 8.85 \\
\hline & Gadus morhua & 20,361 & 7040.85 & 1441.77 & 744.62 & 169.23 & 8.52 \\
\hline & Gasterosteus aculeatus & 26,630 & 6896.85 & 1474.53 & 686.88 & 165.79 & 8.89 \\
\hline & Larimichthys crocea & 21,220 & 9425.27 & 1690.06 & 902.2 & 176.53 & 9.57 \\
\hline & Oreochromis niloticus & 24,562 & 9494.62 & 1789.15 & 829.18 & 173.82 & 10.29 \\
\hline & Oryzias latipes & 23,332 & 8859.46 & 1467.62 & 962.5 & 169.08 & 8.68 \\
\hline & Takifugu rubripes & 19,635 & 7762.47 & 1645.04 & 707.22 & 170.47 & 9.65 \\
\hline \multirow[t]{3}{*}{ De novo } & Augustus & 21,662 & 7149.08 & 1725.00 & 659.42 & 186.98 & 9.23 \\
\hline & Genscan & 25,933 & 9855.53 & 1791.43 & 990.72 & 196.01 & 9.14 \\
\hline & GlimmerHMM & 99,722 & 1192.96 & 594.53 & 378.42 & 230.31 & 2.58 \\
\hline \multirow[t]{2}{*}{ Transcript } & Pasa \& Transdecoder & 33,965 & 4856.71 & 1186.88 & 558.33 & 156.73 & 7.57 \\
\hline & Hisat \& Stringtie & 31,664 & 5551.59 & 1303.52 & 608.39 & 163.3 & 7.98 \\
\hline EVM & & 19,925 & 9418.80 & 1945.48 & 757.65 & 179.08 & 10.86 \\
\hline
\end{tabular}

\begin{tabular}{|ccc|}
\hline Table 8. Statistics of the functional annotation. & \\
\hline Database & Number of genes & Gene functionally annotated (\%) \\
Total & 20,057 & 100.00 \\
SwissProt & 17,333 & 86.42 \\
KEGG & 16,182 & 80.68 \\
TrEMBL & 18,037 & 89.93 \\
Interpro & 17,108 & 85.30 \\
Overall & 18,064 & 90.06 \\
\hline
\end{tabular}

these species were downloaded from NCBI, except for S. schlegelii [46], which was obtained from the China National Genebank Nucleotide Sequence Archive (CNSA; Accession ID: CNP0000222). To improve analysis quality, we removed genes either with frameshifts, or less than 50 amino acids, as well as redundant copies, only keeping the longest transcripts for comparative genomic analysis. A total of 21,022 gene families were identified, of which 40 gene families were unique to the humpback puffer (Table 9 and Figure $5 a$ ).

Of all 21,022 gene families, we identified 4461 single-copy protein-coding genes shared by all species. We used MUSCLE v3.8.31 [47] to align these orthologs, with default parameters. Then, the alignments were concatenated into a 3,584,782 amino acid "super alignment matrix". Based on this matrix, a phylogenetic tree was constructed using RAxML v8.2.4 [48], with the best amino acid substitution model-JTT. Clade support was assessed 


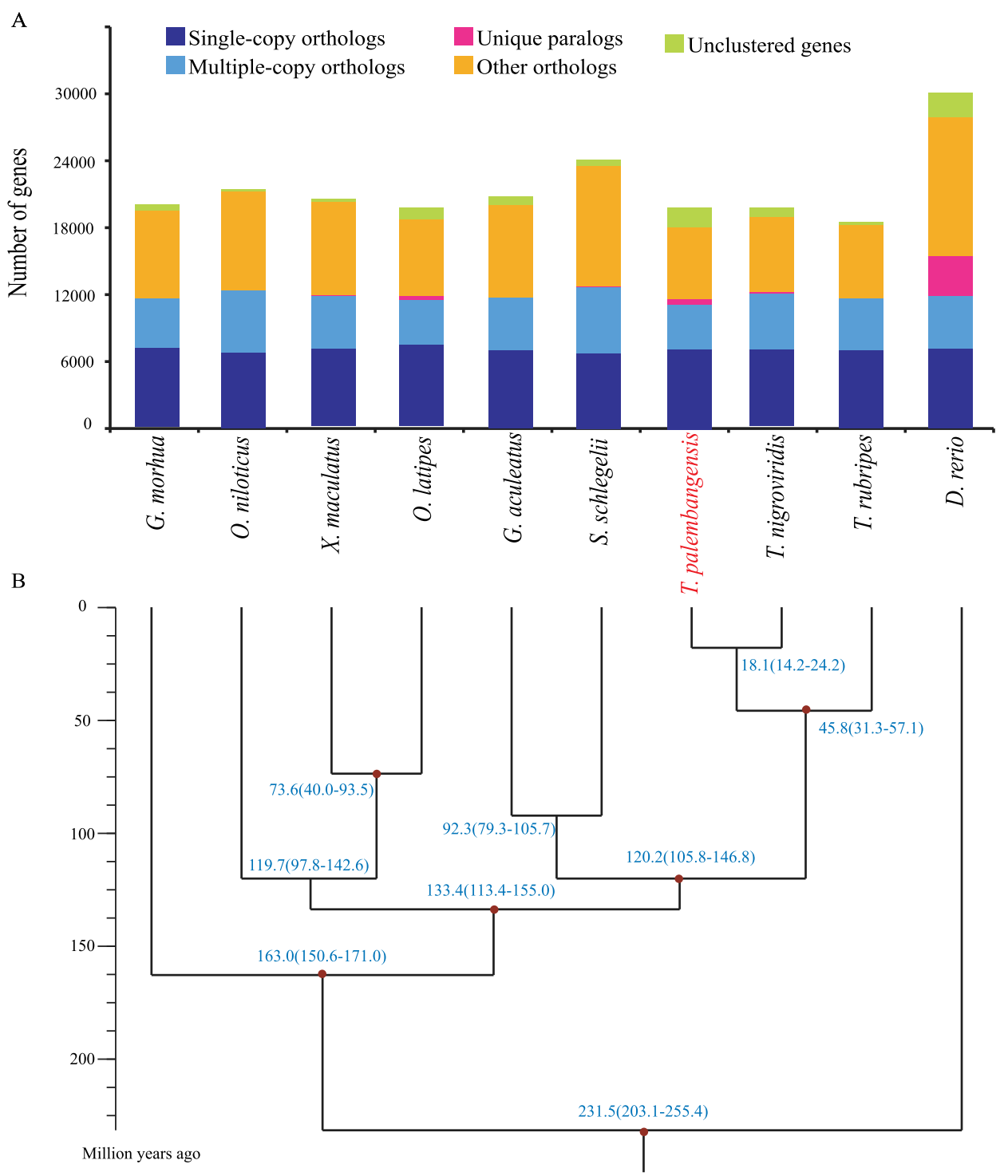

Figure 5. Comparative analysis of the Tetraodon palembangensis and nine teleosts. (a) Clustering of gene families (b) Phylogenetic tree constructed with the single-copy gene families. The fossil correction nodes in the tree are highlighted by red dots.

using a bootstrapping algorithm with 1000 alignment replicates (Figure 5b). Next, we calculated the divergence time among these teleosts using the MCMCTree tool included in PAML (v4.7a, RRID:SCR_014932) [49], with parameters of "-rootage 500 -clock 3 -alpha 0.431879". The fossil correction time (Table 10) was obtained from Timetree [50]. The result showed that the humpback puffer and T. nigroviridis, two species belonging to the same genus, shared a common ancestor 18.1 millon years ago (MYA) and diverged from T. rubripes 18.1 MYA (Figure 5b). 


\begin{tabular}{cccccc}
\hline \multicolumn{2}{l}{ Table 9. Statistics of gene family clustering. } & & & & \\
\hline Species & $\begin{array}{c}\text { Total number } \\
\text { of genes }\end{array}$ & $\begin{array}{c}\text { Number of } \\
\text { unclustered } \\
\text { genes }\end{array}$ & $\begin{array}{c}\text { Number of gene } \\
\text { families }\end{array}$ & $\begin{array}{c}\text { Number of } \\
\text { unique families }\end{array}$ & $\begin{array}{c}\text { Average number } \\
\text { of genes per } \\
\text { family }\end{array}$ \\
D. rerio & 30,067 & 2171 & 18,635 & 735 & 1.5 \\
G. aculeatus & 20,756 & 728 & 15,995 & 11 & 1.25 \\
G. morhua & 19,987 & 525 & 15,650 & 11 & 1.24 \\
S. schlegelii & 24,094 & 558 & 16,991 & 30 & 1.39 \\
O. latipes & 19,535 & 984 & 14,873 & 71 & 1.25 \\
O. niloticus & 21,431 & 160 & 15,811 & 13 & 1.35 \\
T. nigroviridis & 19,544 & 805 & 14,916 & 50 & 1.26 \\
T. palembangensis & 19,796 & 690 & 15,830 & 40 & 1.21 \\
T. rubripes & 18,459 & 207 & 14,733 & 6 & 1.24 \\
X. maculatus & 20,356 & 271 & 16,446 & 3 & 1.22 \\
\hline
\end{tabular}

\begin{tabular}{ccccc}
\hline \multicolumn{6}{l}{ Table 10. Fossil correction time used in divergence analysis. } & & \\
\hline Taxon 1 & Taxon 2 & Fossil time (MYA) & Minimum (MYA) & Maximum (MYA) \\
Takifugu rubripes & Tetraodon nigroviridis & 52 & 42 & 59 \\
Takifugu rubripes & Gadus morhua & 148 & 141 & 170 \\
Oryzias latipes & Xiphophorus maculatus & 93 & 76 & 111 \\
Oryzias latipes & Gasterosteus aculeatus & 128 & 105 & 154 \\
Oryzias latipes & Danio rerio & 229.9 & 204.5 & 255.3 \\
\hline
\end{tabular}

\begin{tabular}{|ccccc|}
\hline Table 11. Statistics of the BUSCO assessment. & & \\
\hline & Genome assembly & \multicolumn{3}{c|}{ Gene set } \\
\hline Types of BUSCOs & Number & Percentage (\%) & Number & Percentage (\%) \\
\hline Complete BUSCOs & 3486 & 95.7 & 3303 & 90.7 \\
Complete and single-copy BUSCOs & 3427 & 94.1 & 3252 & 89.3 \\
Complete and duplicated BUSCOs & 59 & 1.6 & 51 & 1.4 \\
Fragmented BUSCOs & 45 & 1.2 & 96 & 2.6 \\
\hline Missing BUSCOs & 109 & 3.1 & 241 & 6.7 \\
\hline Total BUSCOs groups searched & 3640 & 100 & 3640 & 100 \\
\hline
\end{tabular}

\section{DATA VALIDATION AND QUALITY CONTROL}

To demonstrate the quality of genome assembly and gene set, we performed a qulity evaluation using the actinopterygii_odb10 database from Benchmarking Universal Single-Copy Orthologs (BUSCO v.4.1.2, RRID:SCR_015008) [51]. The results showed that 95.7\% and $90.7 \%$ complete BUSCOs were covered by the genome assembly and gene set, respectively (Table 11).

\section{REUSE POTENTIAL}

We assembled the first annotated chromosome-level genome of the humpback puffer. These resources will be helpful to study the mechanism of body expansion displayed by this fish species, the synthesis mechanism and treatment of tetrodotoxin, as well as the evolution of freshwater puffer. Futhermore, the humpback puffer genome will fill a gap missing from the Fish 10K program and in the phylogenetic tree of life.

\section{DATA AVAILABILITY}

We have deposited the project at CNGB Nucleotide Sequence Archive (CNSA) where the accession ID is CNP0001025. The genomic data can be obtained in GigaScience Database [52]. 
The sequencing data have been deposited at National Center for Biotechnology Information (NCBI) where the bioproject accession ID is PRJNA597275.

\section{DECLARATIONS}

\section{LIST OF ABBREVIATIONS}

bp: base pair; BUSCO: Benchmarking Universal Single-Copy Orthologs; CNSA: China National Gene Bank Nucleotide Sequence Archive; Gb: gigabase; GO: Gene Ontology; kb: kilobase; KEGG: Kyoto Enyclopedia of Genes and Genomes; Mb: megabase; ML: Maximum Likelihood; NCBI: National Center for Biotechnology Information; stLFR: single tube long fragment reads; TE: transposable element.

\section{ETHICAL APPROVAL}

All resources used in this study were approved by the Institutional Review Board of BGI (IRB approval No. FT17007). This experiment has passed the ethics audit of Beijing Genomics Institute (BGI) Gene Bioethics and Biosecurity Review Committee.

\section{CONSENT FOR PUBLICATION}

Not applicable.

\section{COMPETING INTERESTS}

The authors declare that they have no competing interests.

\section{FUNDING}

This work was supported by the special funding of "Blue granary” scientific and technological innovation of China (2018YFD0900301-05).

\section{AUTHORS' CONTRIBUTIONS}

H.Z. and G.F. designed this project. M.Z. prepared the samples. S.L., S.P., W.X., C.W. and C.M. conducted the experiments. R.Z., C.L., M.Y. and X.H. did the analyses. R.Z., C.L. and M.Y. wrote and revised the manuscript. All authors read and approved the final version of the manuscript.

\section{ACKNOWLEDGEMENTS}

We thank for the China National Genebank for technical support in constructing and sequencing the stLFR library.

\section{REFERENCES}

1 Saitanu K et al. Toxicity of the freshwater puffer fish Tetraodon fangi and T. palembangensis from Thailand. Toxicon, 1991; 29: 895-897.

2 Subamia IW, Sudarto S, Purbowasito W, Sex determination in Indonesian pufferfish Tetraodon palembangensis Bleeker, 1852: implication for aquaculture and conservation. Indones. Aquac. J., 2011; 6: $37-45$.

3 Jaillon $\mathbf{O}$ et al. Genome duplication in the teleost fish Tetraodon nigroviridis reveals the early vertebrate proto-karyotype. Nature, 2004; 431: 946-957.

4 Sutaria D, Panicker D, Jog K, Sule M, Muralidharan R, Bopardikar I, Humpback dolphins (Genus Sousa) in India: an overview of status and conservation issues. Adv. Marine Biol., 2015; 72: 229-256.

5 Hedges SB, The origin and evolution of model organisms. Nat. Rev. Genet., 2002; 3: 838-849.

6 Fan G et al. Initial data release and announcement of the 10,000 Fish Genomes Project (Fish10K). GigaScience, 2020; 9: giaa080. 
7 Zhang R et al. Protocols for "Chromosome-level genome assembly of the humpback puffer, Tetraodon palembangensis”. protocols.io. 2021; https://dx.doi.org/10.17504/protocols.io.bs8inhue.

8 Chang L, Hi-C library preparation for the Lateolabrax maculatus genome. protocols.io. 2018l; https://dx.doi.org/10.17504/protocols.io.ss4eegw.

9 Julkowska M, Protocols for “RNA isolation with TRIzol”. protocols.io. 2018; https://dx.doi.org/10.17504/protocols.io.pbndime.

10 Wang $\mathrm{O}$ et al. Efficient and unique cobarcoding of second-generation sequencing reads from long DNA molecules enabling cost-effective and accurate sequencing, haplotyping, and de novo assembly. Genome Res., 2019; 29: 798-808.

11 Huang J et al. BGISEQ-500 WGS library construction. protocols.io. 2018; https://dx.doi.org/10.17504/protocols.io.ps5dng6.

12 Huang J, BGISEQ-500 sequencing. protocols.io. 2018; https://dx.doi.org/10.17504/protocols.io.pq7dmzn.

13 Pederson ER, RNA and DNA extraction (Qiagen) of frozen tissue to nanopore sequencing. protocols.io. 2021; https://dx.doi.org/10.17504/protocols.io.bj7tkrnn.

14 Zhang R, Oxford Nanopore sequencing and library construction. protocols.io. 2021; https://dx.doi.org/10.17504/protocols.io.btignkbw.

15 Chen Y et al. SOAPnuke: a MapReduce acceleration-supported software for integrated quality control and preprocessing of high-throughput sequencing data. Gigascience, 2018; 7: gix120.

16 Servant $\mathrm{N}$ et al. HiC-Pro: an optimized and flexible pipeline for Hi-C data processing. Genome Biol., 2015; 16: 259.

17 Li R et al. The sequence and de novo assembly of the giant panda genome. Nature, 2010; 463: 311-317.

18 Vurture GW et al. GenomeScope: fast reference-free genome profiling from short reads. Bioinformatics, 2017; 33: 2202-2204.

19 Luo R et al. SOAPdenovo2: an empirically improved memory-efficient short-read de novo assembler. Gigascience, 2012; 1: 18.

20 Xu M, Guo L, Gu S, Wang O, Zhang R, Peters BA, Fan G, Liu X, Xu X, Deng L, Zhang Y, TGS-GapCloser: A fast and accurate gap closer for large genomes with low coverage of error-prone long reads. Gigascience, 2020; 9(9): giaa094. doi:10.1093/gigascience/giaa094.

21 Walker BJ et al. Pilon: an integrated tool for comprehensive microbial variant detection and genome assembly improvement. Plos One, 2014; 9: e112963.

22 Liu X, Protocols for "the pipeline of Hi-C assembly". protocols.io. 2018; http://dx.doi.org/10.17504/protocols.io.qradv2e.

23 Wataru K et al. Integration of the genetic map and genome assembly of fugu facilitates insights into distinct features of genome evolution in teleosts and mammals. Genome Biol. Evol., 2011; 3: 424-442.

24 Kang $\mathbf{S}$ et al. Chromosomal-level assembly of Takifugu obscurus (Abe, 1949) genome using third-generation DNA sequencing and Hi-C analysis. Mol. Ecol. Resour., 2020; 20: 520-530.

25 Zhou Y et al. Chromosome genome assembly and annotation of the yellowbelly pufferfish with PacBio and Hi-C sequencing data. Sci. Data, 2019; 6: 267.

26 Mandrioli M, Cuoghi B, Marini M, Manicardi GC, Cytogenetic analysis of the pufferfish Tetraodon fluviatilis (Osteichthyes). Chromosome Res., 2000; 8: 237.

27 Li F et al. Morphological structure and karyotype of Thamnaconus septentrionalis. South China Fisheries Science, 2019; 15: 104-112.

28 Benson G, Tandem repeats finder: a program to analyze DNA sequences. Nucleic Acids Res., 1999; 27: 573-580.

29 Tarailo-Graovac M, Chen N, Using RepeatMasker to identify repetitive elements in genomic sequences. Curr. Protoc. Bioinformatics, 2009; 25: 4.10.1-4.10.14.

30 Meng G, Li Y, Yang C, Liu S, MitoZ: a toolkit for animal mitochondrial genome assembly, annotation and visualization. Nucleic Acids Res., 2019; 47: e63.

31 Wataru I et al. MitoFish and MitoAnnotator: a mitochondrial genome database of fish with an accurate and automatic annotation pipeline. Mol. Biol. Evol., 2013; 30: 2531-2540.

32 Stanke M, Keller O, Gunduz I, Hayes A, Waack S, Morgenstern B, AUGUSTUS: ab initio prediction of alternative transcripts. Nucleic Acids Res., 2006; 34(suppl_2): W435-W439. 
33 Majoros WH, Pertea M, Salzberg SL, TigrScan and GlimmerHMM: two open source ab initio eukaryotic gene-finders. Bioinformatics, 2004; 20: 2878-2879.

34 Burge C, Karlin S, Prediction of complete gene structures in human genomic DNA. J. Mol. Biol., 1997; 268: 78-94.

35 Grabherr MG et al. Full-length transcriptome assembly from RNA-Seq data without a reference genome. Nat. Biotechnol., 2011; 29: 644.

36 Pertea M, Kim D, Pertea GM, Leek JT, Salzberg SL, Transcript-level expression analysis of RNA-seq experiments with HISAT, StringTie and Ballgown. Nat. Protoc., 2016; 11: 1650.

37 Campbell MA, Haas BJ, Hamilton JP, Mount SM, Buell CR, Comprehensive analysis of alternative splicing in rice and comparative analyses with Arabidopsis. BMC Genomics, 2006; 7: 327.

38 Doerks T, Copley RR, Schultz J, Ponting CP, Bork P, Systematic identification of novel protein domain families associated with nuclear functions. Genome Res., 2002; 12: 47-56.

39 Haas BJ et al. Automated eukaryotic gene structure annotation using EVidenceModeler and the Program to Assemble Spliced Alignments. Genome Biol., 2008; 9: R7.

40 Elsik CG, Mackey AJ, Reese JT, Milshina NV, Roos DS, Weinstock GM, Creating a honey bee consensus gene set. Genome Biol., 2007; 8: R13.

41 Bairoch A, Apweiler R, The SWISS-PROT protein sequence database and its supplement TrEMBL in 2000. Nucleic Acids Res., 2000; 28: 45-48.

42 Kanehisa M, Goto S, KEGG: kyoto encyclopedia of genes and genomes. Nucleic Acids Res., 2000; 28: 27-30.

43 Harris MA, Clark J, Ireland A, Lomax J, Ashburner M, Foulger R, The Gene Ontology (GO) database and informatics resource. Nucleic Acids Res., 2004; 32(Suppl_1): D258-D261.

44 Jones P et al. InterProScan 5: genome-scale protein function classification. Bioinformatics, 2014; 30 : 1236-1240.

45 Li H et al. TreeFam: a curated database of phylogenetic trees of animal gene families. Nucleic Acids Res., 2006; 34: D572.

46 He Y et al. A chromosome level genome of black rockfish, Sebastes schlegelii, provides insights into the evolution of live birth. Mol. Ecol. Resour., 2019; 19: 1309-1321.

47 Edgar RC, MUSCLE: multiple sequence alignment with high accuracy and high throughput. Nucleic Acids Res., 2004; 19(32): 1792-1797.

48 Stamatakis A, RAxML-VI-HPC: Maximum likelihood-based phylogenetic analyses with thousands of taxa and mixed models. Bioinformatics, 2006; 22: 2688-2690.

49 Yang Z, PAML: a program package for phylogenetic analysis by maximum likelihood. Bioinformatics, 1997; 13: 555-556.

50 Sudhir K, Stecher G, Suleski M, Hedges SB, TimeTree: a resource for timelines, timetrees, and divergence times. Mol. Biol. Evol., 2017; 34: 1812.

51 Simão FA, Waterhouse RM, Ioannidis P, Kriventseva EV, Zdobnov EM, BUSCO: assessing genome assembly and annotation completeness with single-copy orthologs. Bioinformatics, 2015; 31: 3210-3212.

52 Zhang R et al. Genome data for the chromosome-level assembly of the humpback puffer, Tetraodon palembangensis. GigaScience Database. 2020; http://dx.doi.org/10.5524/100755. 